\title{
Characteristics of droplet motion in effervescent sprays
}

\author{
Jan Jedelský ${ }^{1, a}$, Matouš Zaremba ${ }^{1}$, Milan Malý ${ }^{1}$ and Miroslav Jícha ${ }^{1}$ \\ ${ }^{1}$ Brno University of Technology, Faculty of Mechanical Engineering, Energy Institute, Technická 2896/2, 61669 Brno, \\ Czech Republic
}

\begin{abstract}
Time resolved droplet size and velocity measurement was made using Phase-Doppler anemometry in an effervescent spray at GLR of $6 \%$ and operation pressure drops $21-52 \mathrm{kPa}$. The spray shows a size dependent variation of mean as well as fluctuating axial and radial velocities of droplets similarly for all operation regimes. Particles under $13 \mu \mathrm{m}$ follow the gas flow, axially decelerated due to gas expansion. Velocity of medium sized particles is positively size correlated and larger particles keep high velocity, given them during discharge. Fluctuating radial velocity of small particles is larger than that of large particles while fluctuating axial velocity increases with size. Small particles thus reach a ratio of radial to axial velocity fluctuations $\sim 0.6$ but large particles only $\sim 0.1$, which indicates large transverse dispersion of small particles. Overall fluctuating velocity ratios smaller than 0.5 document an anisotropic character of the liquid mass fluctuations. Power spectral density (PSD) of axial velocity fluctuations of large droplets is uniform up to $1 \mathrm{kHz}$, while PSD of smaller particles drops down with frequency for frequencies $>100 \mathrm{~Hz}$. Large particles thus preserve the fluctuations imposed during discharge while the gas turbulence drops with frequency. Turbulence intensity reaches 14 to $21 \%$ depending on pressure. Such high-turbulence character of the flow probably results from a heterogeneous gas-liquid mixture at the discharge.
\end{abstract}

\section{Introduction}

Twin-fluid atomizers with internal mixing, also referred as pneumatic or air-assist atomizers, have a potential to replace pressure atomizers in a number of applications. Their operation is based on the mixing of a gas (usually air or steam, based on their availability) with liquid to be atomized inside a chamber of the atomizer, followed with a discharge of the created two-phase gas-liquid mixture, its primary breakup, secondary breakup of formed droplets [1] and possible droplet collisions.

Formation and development of the spray of internallymixed atomizers is widely studied in past twenty five years [2-6], however more detailed insight into the development of the spray and into droplet dynamics is required to fully benefit from the potentialities of twinfluid atomization technique.

We can describe the spray formation according these previous works as follows. The atomizing gas is injected with low relative velocity into the liquid moving through the central tube of the atomizer (see Figure 2). Gas-liquid two-phase flow is created in this tube prior the atomizer discharge orifice. It can be bubbly, foam or dispersed depending on the mixing ratios of the gas and liquid [7]. The liquid, when reaches the discharge orifice, is disintegrated by the coo-flowing gas stream into a film and ligaments. Gas volumes emerging from the nozzle at given pressure drop (tens of $\mathrm{kPa}$ here), expand rapidly and transform the liquid into droplets. Interaction between the two fluids depends significantly on relative amount of gas to liquid in the mixture, GLR.

Gas is discharged with higher velocity than liquid and accelerates the liquid volumes near the nozzle. The liquid breaks up into smaller fractions that are more receptive to the gas drag force. The expanding gas decelerates with distance from the orifice and in certain distance its velocity drops below the liquid velocity. This distance however depends on operation conditions and namely on the size of liquid fragments - droplets. It results in a correlation between droplet size and their velocity, which can be positive, negative as well as more complicated depending on the position in the spray and on the operation conditions.

We have already investigated the process of energy transfer in effervescent atomization [8] which shows, that typical atomization efficiency is in fragments of per cent. In another paper [9] we analyse a distribution of liquid mass within the effervescent spray and its temporal variation using combined PIV-PLF technique. In this paper we address the issue of a size-dependent droplet motion in the spray generated by a single-hole effervescent atomizer. Droplet dynamics is described using experimental data acquired by Phase-Doppler anemometry.

\footnotetext{
a Corresponding author: jedelsky@fme.vutbr.cz
} 


\section{Experimental equipment and methods}

All the data, included in this work, were acquired by cold testing of an effervescent atomizer in the Spray laboratory at Energy department, Faculty of Mechanical Engineering, Brno University of Technology. The test equipment consists of a test bench with fluid supply system, a Phase-Doppler Analyzer (PDA) and an effervescent atomizer.

\subsection{Test bench}

The atomizer is operated on a test bench (Figure 3), which is equipped with fuel and air supplies (Figure 1). The fuel supply system consists of two circuits; one circuit enables fuel temperature variation using a chiller and a heat exchanger, the second circuit pushes the fuel into the nozzle using gear pump. This circuit also contains a filter and Siemens Mass 2100 Coriolis flow meter fitted with Mass 6000 Ex transmitter. Regulation valves and a bypass with a regulation valve are used for precise regulation of flow-rate.

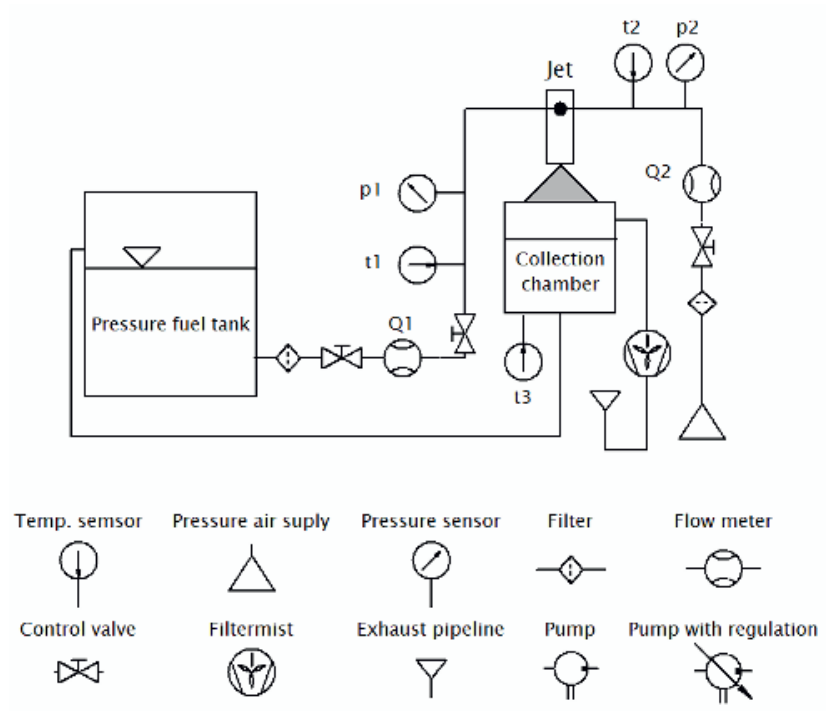

Figure 1. Schematic layout of the test bench [10].

Air is taken from the central pressure-air system. Maximum pressure is $0.8 \mathrm{MPa}$. Air flows through a dehumidifier and a filter into regulation valve. Both lines are equipped with pressure sensors (BD sensors DMP 33li) and thermometers (resistance temperature sensor Omega SPRTX-S1).

\subsection{Atomizer}

An effervescent atomizer with the "outside-in" gas injection configuration is investigated. Atomized liquid, light heating oil (abbrev. LHO), is supplied into a mixing chamber through central orifice. Density of the oil is $874 \mathrm{~kg} \cdot \mathrm{m}^{-3}$ and dynamic viscosity is $0.0185 \mathrm{~kg} \cdot \mathrm{m}^{-1} \cdot \mathrm{s}^{-1}$ at $20{ }^{\circ} \mathrm{C}$. The oil is used as a representation of hydrocarbon fuels and allows for realistic atomization results, contrary to water which is often used in laboratory experiments and which has different rheological properties compared to hydrocarbon liquids [11]. Atomizing gas (air at temperature of $20^{\circ} \mathrm{C}$ ) is injected into the mixing chamber through 24 aeration holes $1 \mathrm{~mm}$ in diameter (Figure 2).

Two-phase mixture, formed by the liquid and gas mixing, is pushed towards the exit orifice of the atomizer. The mixture expands rapidly into ambient air after it passes through the exit orifice with a diameter of $1 \mathrm{~mm}$. The atomizer was operated in downward vertical position and the mixture was discharged into still ambient air at barometric pressure.

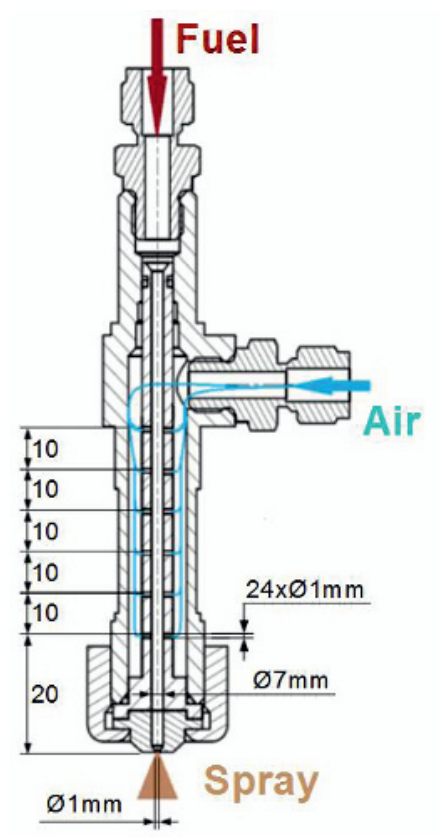

Figure 2. Tested nozzle.

Vertical axis, $z$, corresponds to the main atomizer axis and to the axial velocity component as measured using PDA. Origin of the coordinate system is identical to the exit orifice centre. The $x$-axis and $y$-axis are placed in the horizontal plane. The $x$-axis corresponds to the radial velocity component.

\subsection{Phase-Doppler analyser}

2D Fiber based PDA system by Dantec Dynamics (Figure 3 and 4), was used for measurements of time resolved droplet size and two velocity components (in axial and radial direction of the nozzle main axis). The system consists of:

- Spectra physics Stabilite 2017 Argon laser: max. power output 6 Watts,

- 60X41 Transmitter, which splits the beam into its individual colour components $(488.0 \mathrm{~nm}, 514.5 \mathrm{~nm})$ and divides each colour into two beams.

- Brag cell is implemented in the transmitter which gives frequency shift $40 \mathrm{MHz}$ to each beam from given pair.

- 60X81 2D $85 \mathrm{~mm}$ transmitting optics with 50X82 beam translator,

- 57X50 $112 \mathrm{~mm}$ diameter fibre PDA receiver optics with spatial filter,

- Fiber PDA Detector unit and

- BSA P80 flow and particle processor. 


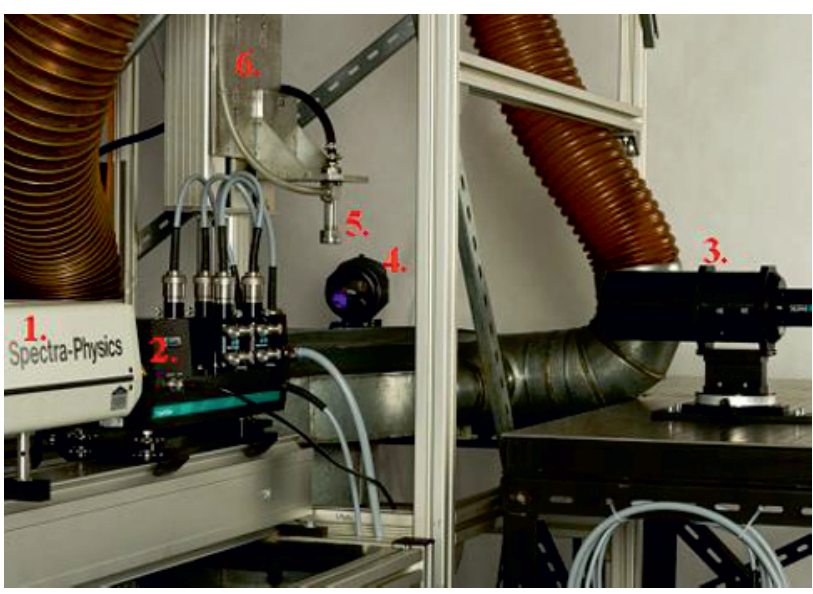

Figure 3. Photograph of the test bench with PDA system. (1- Ar-Ion Laser, 2- Transmitter, 3-Transmitting optics, 4Receiving optics, 5-Nozzle, 6-Traversing system).

Focal lengths were chosen $500 \mathrm{~mm}$ for the transmitting optics and $800 \mathrm{~mm}$ for the receiving optics. The half-intersection angle between the laser beams was se to $4.303^{\circ}$ for separation of the beams of $60 \mathrm{~mm}$. Dimensions of the measurement volume are $d_{x}=0.1166 \mathrm{~mm}, d_{y}=0.1162 \mathrm{~mm}$ and $d_{z}=1.549 \mathrm{~mm}$. Slit size of $0.05 \mathrm{~mm}$ was used to reduce the $d_{z}$ dimension of the measurement volume. The scattering angle (angle between the transmitting and the receiving optics) was set to $69^{\circ}$ (Brewster's angle condition to avoid reflected light). Every measurement was done for 50,000 particles, which is statistically sufficient number for estimation of characteristic values of the spray (diameters and velocities) as well as it gives long enough sample for sufficiently well frequency resolved spectral analysis of velocity fluctuations.

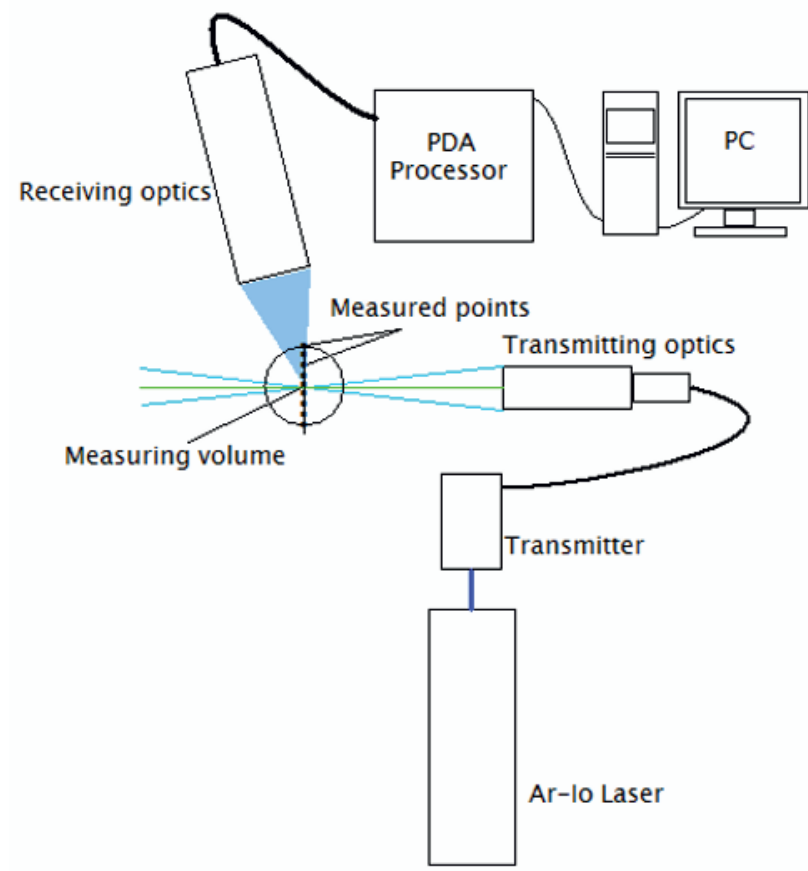

Figure 4. PDA schematic layout [10].

\section{Results}

Measurement of droplet size and velocity was made in one spray position, $50 \mathrm{~mm}$ downstream the exit orifice in the nozzle axis. Spray in this position can be characterised as fully developed [12], but it is not yet significantly affected by droplet collisions, gravity force, mist exhaust flow and other parasite effects. This distance is also a typical position of flame zone in the case of reacting sprays (combustion applications). The measurement was done for four operation regimes of the atomizer, see Table 1. Operation point of effervescent atomizers is represented by pressure drop $p$ on the atomizer available for the atomization (difference between gas inlet pressure and ambient - barometric pressure) and by GLR (ratio of gas mass flow-rate to the liquid mass flow-rate).

Table 1. Operation conditions of the atomizer.

\begin{tabular}{|c|c|c|c|}
\hline \multirow{2}{*}{$\begin{array}{c}\boldsymbol{p} \\
(\mathbf{k P a})\end{array}$} & $\mathbf{G L R}$ & \multicolumn{2}{|c|}{ Flow-rate (kg/hour) } \\
\cline { 3 - 4 } & $\mathbf{\%})$ & LHO & Air \\
\hline 21 & 6.2 & 1.61 & 0.10 \\
\hline 32 & 6.3 & 2.14 & 0.14 \\
\hline 41 & 6.1 & 2.43 & 0.15 \\
\hline 52 & 6.4 & 2.72 & 0.17 \\
\hline
\end{tabular}

\subsection{Mean and fluctuating droplet velocities}

Liquid breaks up into droplets with approximately lognormal size distribution, as shown in Figure 5, top. Maximum droplet frequency is found for droplet size of $12 \mu \mathrm{m}$, very low number of droplets is smaller than $1 \mu \mathrm{m}$ or larger than $80 \mu \mathrm{m}$ for nozzle operation at $p=52 \mathrm{kPa}$ and GLR $=6.4 \%$. Sauter mean diameter of droplets, $D_{32}$, is about $59 \mu \mathrm{m}$. Similar size distribution would be found at other regimes, the particle size increases with decrease in the pressure drop, in accordance with previous works [1], as documented in Table 2. Figure 5, bottom illustrates a correlation between axial and radial velocity of individual droplets for three size classes. About 1,000 droplets in each class are displayed only for good readability. The data were classified into size bins with $d_{p \max } / d_{p \min }=\sqrt{2}$ in order to resolve the effect of droplet size on their dynamics. Mean and fluctuating (root-mean-square, RMS) axial as well as radial velocities for each size class were evaluated, see Figure 6.

Table 2. Results of PDA measurements.

\begin{tabular}{|c|c|c|c|c|c|}
\hline $\begin{array}{c}\boldsymbol{p} \\
(\mathbf{k P a})\end{array}$ & $\begin{array}{c}\boldsymbol{D}_{32} \\
(\boldsymbol{\mu \mathbf { m } )}\end{array}$ & $\begin{array}{c}\bar{v}_{x} \\
(\mathbf{m} / \mathbf{s})\end{array}$ & $\begin{array}{c}\bar{v}_{z} \\
(\mathbf{m} / \mathbf{s})\end{array}$ & $\begin{array}{c}\boldsymbol{k} \\
\left(\mathbf{m}^{\mathbf{2}} \cdot \mathbf{s}^{-\mathbf{2}}\right)\end{array}$ & $\begin{array}{c}\boldsymbol{I} \\
(\mathbf{\%})\end{array}$ \\
\hline 21 & 117 & 5.93 & -0.04 & 2.46 & 21.2 \\
\hline 32 & 73 & 8.60 & 0.01 & 2.88 & 16.9 \\
\hline 41 & 67 & 8.99 & -0.14 & 3.37 & 16.9 \\
\hline 52 & 59 & 13.1 & -0.09 & 5.14 & 14.3 \\
\hline
\end{tabular}



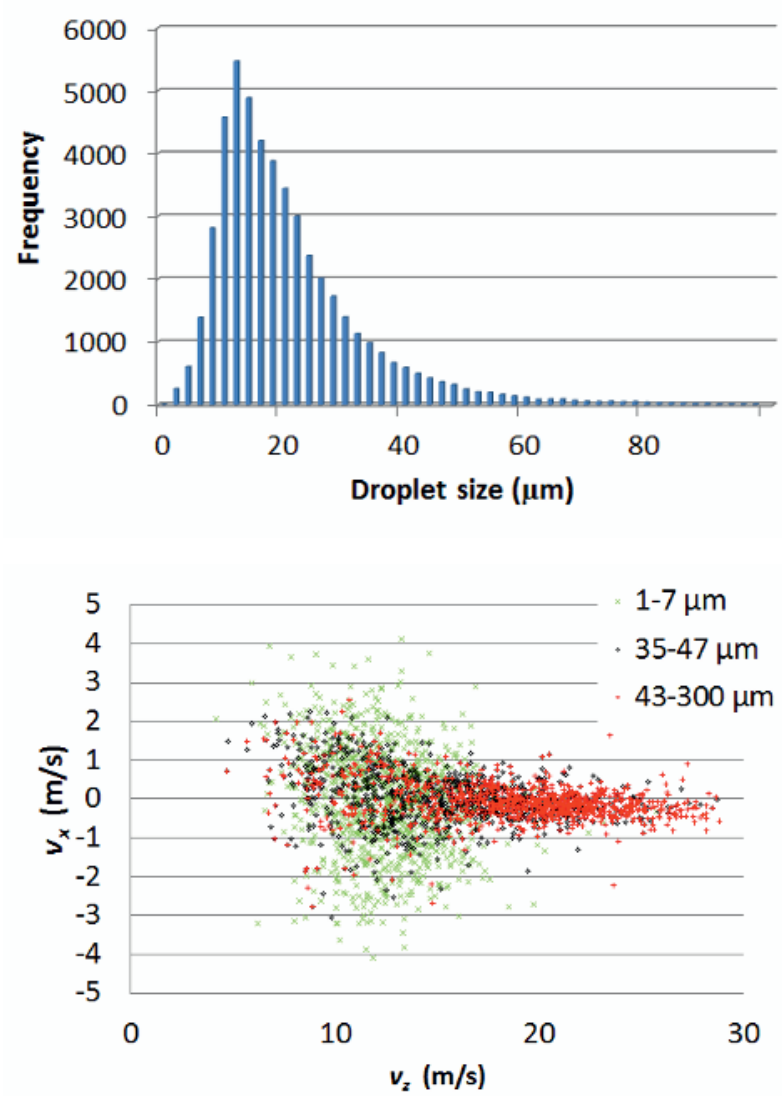

Figure 5. Droplet size spectra (top), correlation between axial and radial velocity for three size classes (bottom), $p=52 \mathrm{kPa}$, GLR $=6.4 \%$.

Temporally mean axial velocity, $\bar{v}_{z}$, varies significantly with size (Figure 6, top). Small enough particles $\left(d_{p}<13 \mu \mathrm{m}\right)$ follow the gas flow, which already decelerate bellow the original particle velocity at the position of $50 \mathrm{~mm}$ downstream the exit orifice. These small particles decelerate according the gas to $\bar{v}_{z} \sim 12.5 \mathrm{~m} / \mathrm{s}$ for nozzle operation at $p=52 \mathrm{kPa}$ as shown in the Figure 6, top. Particles with $13<d_{p}<75 \mu \mathrm{m}$ have their velocity positively correlated with size, due to their weakening reaction to the drag force with their diameter and particles larger than $75 \mu \mathrm{m}$ keep high axial velocity $\sim 22 \mathrm{~m} / \mathrm{s}$, given them during discharge. Mean radial velocity, $\bar{v}_{x}$, is effectively zero for all size classes due to axial symmetry of the spray and it is therefore not documented graphically in the paper.

Fluctuating axial velocity, $v_{z}^{\prime}$, reaches $2.5-4.6 \mathrm{~m} / \mathrm{s}$, depending on particle size (Figure 6, middle). Fluctuating radial velocity, $v_{x}^{\prime}$, is $\sim 1.3 \mathrm{~m} / \mathrm{s}$ for particles smaller than $10 \mu \mathrm{m}$ and for larger particles gradually decreases with their size (Figure 6, bottom). Small particles thus reach $v_{x}^{\prime} / v_{z}^{\prime} \sim 0.6$ but large particles reach $v_{x}^{\prime} / v_{z}^{\prime}$ only about 0.1. Small particles therefore undergo significant transverse dispersion while large particles move primarily in axial direction. The particles with $d_{p}<13 \mu \mathrm{m}$ represent about $5 \%$ of total droplet mass and particles with $d_{p}<75 \mu \mathrm{m}$ about $50 \%$ of total droplet mass for the nozzle operation at $p=52 \mathrm{kPa}$. It means that the transverse turbulent motion can have some effect for example in combustion applications as it partially controls the mass transfer and leads to a dispersion of the fine droplets. It increases the spatial uniformity of air to liquid ratio, $\lambda$, which consequently improves combustion, homogenizes temperature field and reduces emissions of $\mathrm{NO}_{\mathrm{x}}$ (reduced temperature peaks) and $\mathrm{C}_{\mathrm{m}} \mathrm{H}_{\mathrm{n}}$ (lower number of areas with $\lambda<1$ ).

The $v_{x}^{\prime} / v_{z}^{\prime}$ values are smaller than 0.5 for the droplet set containing all the particle sizes, which indicates anisotropic overall character of the liquid mass fluctuations. The turbulence (fluctuations of gas and small particles) is generally anisotropic as well, with major part of turbulence kinetic energy (TKE) contained preferably in axial direction of the flow, but it is less than for the liquid mass.
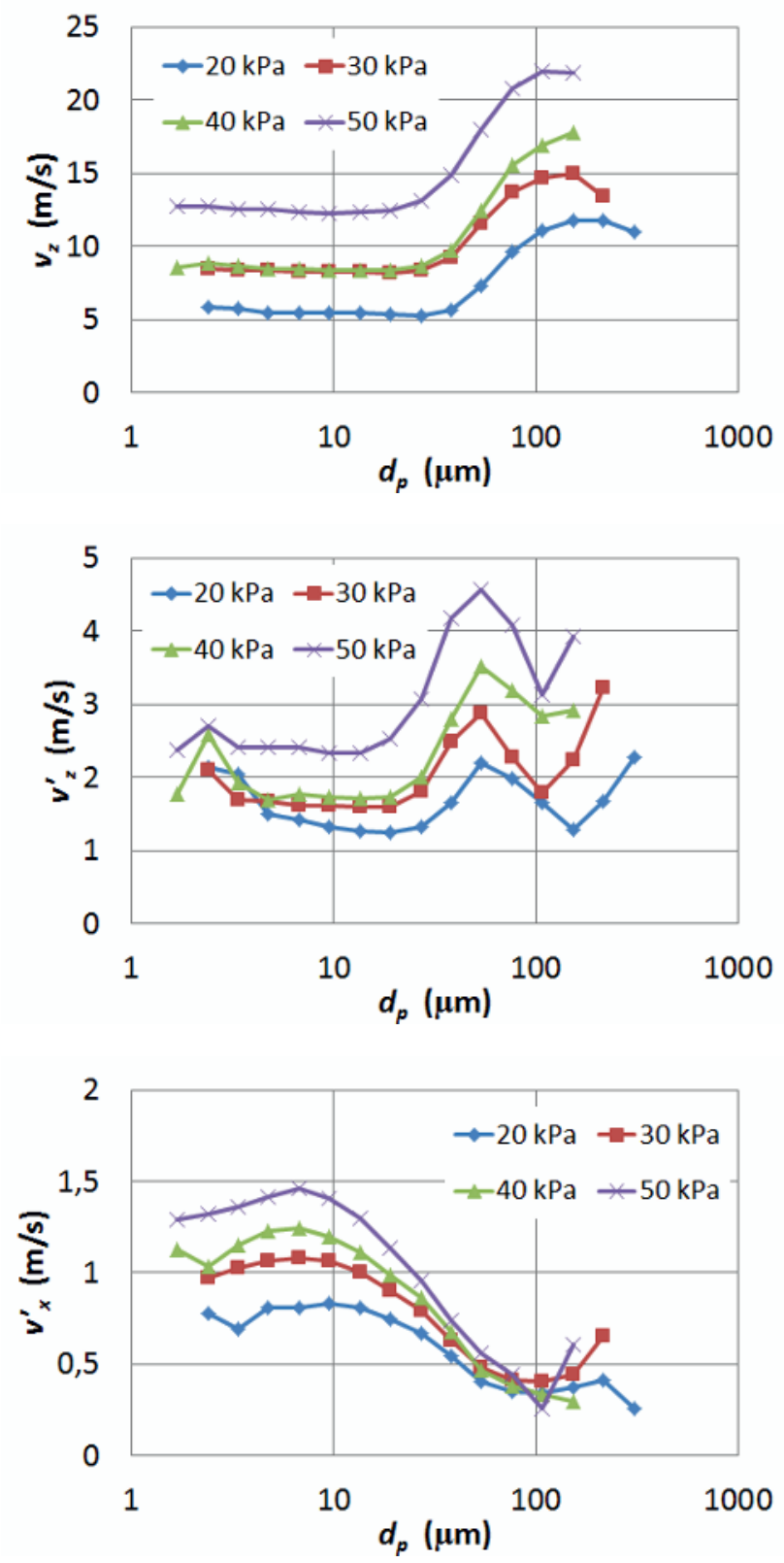

Figure 6. Mean axial velocity (top), fluctuating axial velocity (middle), fluctuating radial velocity (bottom), pressure drop varying, GLR $=6 \%$. 
Similar behaviour as described for the nozzle operation at $p=52 \mathrm{kPa}$ is seen in other pressure conditions, 21,32 and $41 \mathrm{kPa}$, respectively. Velocity magnitudes moreover depend on the pressure drop, $p$. Mean axial velocity uniformly increases with $p$ as it controls the input energy of both fluids and therefore the exit velocity. Fluctuating axial velocity shows similar trends for particles larger than $10 \mu \mathrm{m}$ with pressure depending magnitude. But smaller, gas driven, particles fluctuate with similar velocity in the axial direction at all regimes. Radial fluctuations profiles (Figure 6, bottom) have similar shape for all the pressure conditions as well, but gradual increase of $v_{x}^{\prime}$ with $p$ appears for small particles and very similar $v_{x}^{\prime}$ values are found for large particles conversely. It suggests that original $v_{x}^{\prime}$ of the liquid mass at the nozzle exit, which conserves downstream for large particles, is very similar for different $p$ values. And the discharge $v_{x}^{\prime}$ of the gas phase or differs from that of the liquid or a part of the $v_{z}^{\prime}$ transfers into $v_{x}^{\prime}$ during the flow.

\subsection{Frequency spectra of velocity fluctuations}

Power spectral density (PSD) of velocity fluctuations was calculated using the PDA data. Each data set was divided into three drop-size classes each containing 16,384 samples for spectral analysis. Frequency spectra of PSD for the three size classes, with average size of 9, 19 and $35 \mu \mathrm{m}$ respectively, are shown in Figure 7 separately for axial (top) and radial (bottom) fluctuating velocity. PSD of axial velocity fluctuations of the largest droplets is relatively uniform up to the highest estimated frequency, $1 \mathrm{kHz}$, while PSD of smaller particles tend to drop down with frequency for frequencies larger than $100 \mathrm{~Hz}$. It suggests that large particles preserve the fluctuations imposed during discharge while the gas turbulent motion (and also smaller particles that are strongly influenced by the flow) show a decay of fluctuations with frequency. Also the amplitude of the fluctuations varies with the size; large particles fluctuate more strongly than medium and small ones in axial direction and vice versa in radial direction due to above described reasons.

\subsection{Turbulence kinetic energy}

Turbulence kinetic energy, $k$, can be estimated using the droplet velocity data as

$$
k=\frac{1}{2}\left[\overline{\left(v_{x}^{\prime}\right)^{2}}+\overline{\left(v_{y}^{\prime}\right)^{2}}+\overline{\left(v_{z}^{\prime}\right)^{2}}\right] .
$$

where $v_{x}^{\prime}, v_{y}^{\prime}$ and $v_{z}^{\prime}$ are the velocity fluctuations along corresponding axes, while the overbars represent ensemble averages. We assume, due to the spray axial symmetry, that $v_{x}^{\prime} \cong v_{y}^{\prime}$ and these velocity components are independent (not correlated) and thus $k \cong 0.5\left[2 \overline{\left(v_{x}^{\prime}\right)^{2}}+\overline{\left(v_{z}^{\prime}\right)^{2}}\right]$ and these gas fluctuating velocity components $v_{x}^{\prime}$ and $v_{y}^{\prime}$ are estimated from corresponding droplet velocities for particles with Stokes number $<<1$.

Turbulence intensity is given as

$$
I=\frac{\sqrt{\frac{2}{3} k}}{\bar{v}},
$$

where $\bar{v}$ is mean velocity and in our case $\bar{v} \cong \bar{v}_{z}$.

Table 2 shows that $k$ increases with $p$ as a consequence of increasing Reynolds number in the exit orifice. Turbulence intensity values, $I$, are relatively large, so such flow represents a high-turbulence case. The gas escapes from the nozzle as a part of two-phase mixture. Internal flow has bubbly or foam character in our cases [8] and its heterogeneity preserves during discharge. It is probably the main source of velocity fluctuations in the spray, as already found in [13] and [7] and it is the reason for such large turbulence intensity. This flow character should be taken into account in applications such as liquid combustion, impact cooling and others where unsteady mass transfer has important consequences.
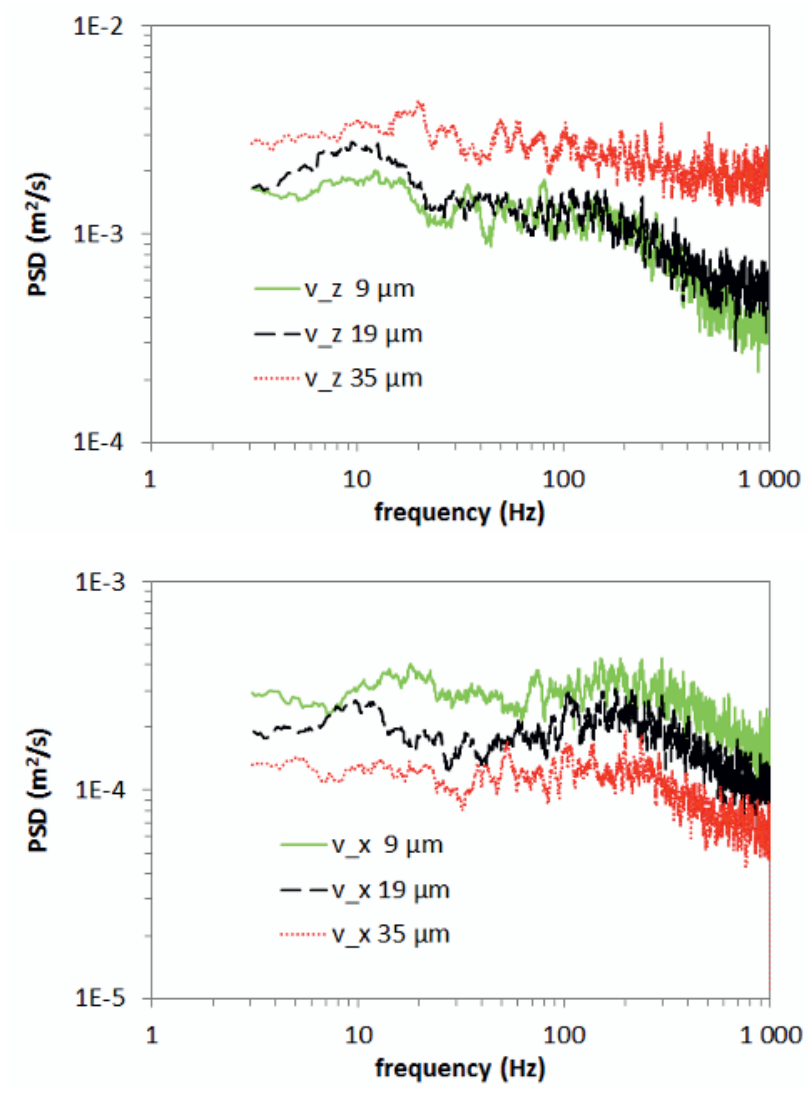

Figure 7. PSD of axial (top) and radial (bottom) velocity fluctuations for different size classes, $p=52 \mathrm{kPa}, \mathrm{GLR}=6.4 \%$.

\section{Conclusions}

Time resolved measurement of droplet size and velocity was made using PDA in an effervescent spray in position $50 \mathrm{~mm}$ downstream the exit orifice in the nozzle axis for four operation regimes of the atomizer at varying pressure drop, $p$, at the atomizer. 
The droplets represent approximately log-normal size distribution with Sauter mean diameter of $117 \mu \mathrm{m}$ at $p=21 \mathrm{kPa}$ and decreasing to $59 \mu \mathrm{m}$ at $p=52 \mathrm{kPa}$. The spray shows a size dependent variation of mean as well as fluctuating axial and radial velocities of droplets. Character of these variations is similar for all regimes.

Small particles $\left(d_{p}<13 \mu \mathrm{m}\right)$ follow the gas flow, which decelerate in axial direction already bellow the discharge particle velocity due to gas expansion. Velocity of particles with $13<d_{p}<75 \mu \mathrm{m}$ is positively correlated with their size and larger particles keep high velocity, given them during discharge. Mean radial velocity is approximately zero for all size classes due to spray axial symmetry.

Fluctuating radial velocity $v_{x}^{\prime}$ of particles smaller than $10 \mu \mathrm{m}$ is relatively large and for larger particles it gradually decreases with their size while fluctuating axial velocity, $v_{z}^{\prime}$, generally increases with size. Small particles thus reach $v_{x}^{\prime} / v_{z}^{\prime} \sim 0.6$ but large particles reach $v_{x}^{\prime} / v_{z}^{\prime}$ only about 0.1 . This significant transverse dispersion of small particles can have some effect for example in combustion applications as it partially controls the mass transfer. The $v_{x}^{\prime} / v_{z}^{\prime}$ values are smaller than 0.5 for whole size data set, which indicates anisotropic overall character of the liquid mass fluctuations. The results moreover show that $v_{x}^{\prime}$ of the liquid mass at the nozzle exit conserves downstream for large particles and that the discharge $v_{x}^{\prime}$ of the gas phase or differs from the $v_{x}^{\prime}$ of the liquid or a part of the $v_{z}^{\prime}$ transfers into $v_{x}^{\prime}$ during the flow.

Power spectral density of axial velocity fluctuations of the largest droplets is relatively uniform up to $1 \mathrm{kHz}$, while PSD of smaller particles tend to drop down with frequency for frequencies larger than $100 \mathrm{~Hz}$. It suggests that large particles preserve the fluctuations imposed during discharge while the gas turbulent motion decays with frequency.

Turbulence intensity, as estimated using the droplet velocity data, reaches 14 to $21 \%$ depending on $p$ which indicates a high-turbulence character of the flow.
It is probably caused by a discharge of heterogeneous gas-liquid mixture.

\section{Acknowledgements}

Authors greatly acknowledge financial support from project No. 101/11/1264 funded by the Czech Grant Agency and from Operational Programme "Research and Development for Innovations" - "NETME Centre - New Technologies for Mechanical Engineering" Reg. No. CZ.1.05/2.1.00/01.0002.

\section{References}

1. S. D. Sovani, P. E. Sojka, and A. H. Lefebvre, Progress in Energy and Combustion Science 27 (4), 483 (2001).

2. H. Lefebvre, X. F. Wang, and C. A. Martin, Journal of Propulsion and Power 4 (4), 293 (1988).

3. H. Lefebvre, Particle \& Particle Systems Characterization 13 (3), 205 (1996).

4. M. T. Lund and P. E. Sojka, in Proc. ILASS-Americas 1992 (1992), pp. 233.

5. M. V. Panchagnula and P. E. Sojka, Fuel 78 (6), 729 (1999).

6. J. Schroder, A. Gunther, K.-E. Wirth, H. P. Schuchmann, and V. Gaukel, 23 (1), 1 (2013).

7. J. Jedelsky and M. Jicha, Atomization and Sprays 18 (1), 49 (2008).

8. J. Jedelsky and M. Jicha, Fuel 111 (0), 836 (2013).

9. J. Jedelsky and M. Jicha, Atomization and Sprays 22 (7), 603 (2012).

10. M. Zaremba, Brno University of Technology, 2013.

11. H. C. Simmons and C. F. Harding, Journal of Engineering for Power-Transactions of the Asme 103 (1), 118 (1981).

12. J. D. Whitlow and A. H. Lefebvre, Atomization and Sprays 3 (2), 137 (1993).

13. J. T. K. Luong and P. E. Sojka, Atomization and Sprays 9 (1), 87 (1999). 